\title{
LA PERCEPCIÓN DE LOS PADRES SOBRE LA CRIANZA DE LOS HIJOS
}

\author{
Mirna García-Méndez ${ }^{1 *}$, Sofía Rivera Aragón ${ }^{1}$, Isabel Reyes-Lagunes ${ }^{1}$ \\ ${ }^{1}$ Universidad Nacional Autónoma de México
}

Recibido, octubre 22/2013

Concepto de evaluación, junio 5/2014

Aceptado, junio 30/2014
Referencia: García-Méndez, M., Rivera Aragón, S. \& Reyes-Lagunes, I. (2014). La percepción de los padres sobre la crianza de los hijos. Acta Colombiana de Psicología, 17 (2), pp. 133-141. DOI:10.14718/ ACP.2014.17.2.14

Resumen

El objetivo del estudio fue elaborar una escala para evaluar los estilos de crianza utilizados por padres y madres. La muestra fue no probabilística y estuvo conformada por 790 participantes, 350 padres y 440 madres de la ciudad de México, con un promedio de edad de 36.34 años. La edad de los hijos fue de 6 a 12 años. Se elaboró una escala Likert con seis opciones de respuesta (totalmente en desacuerdo-totalmente de acuerdo). Los participantes respondieron la escala de manera voluntaria. Después de realizar el análisis psicométrico a los reactivos (sesgo, correlación ítem total, $t$ de Student para grupos extremos, análisis factorial de componentes principales con rotación ortogonal, alpha de Cronbach) la escala quedó integrada por 24 reactivos distribuidos en cinco factores: castigo, permisivo, emocional negativo, control conductual, y cognición negativa. Se concluye que se obtuvo una escala válida y confiable para evaluar los estilos de crianza empleados por padres y madres. Palabras clave: hijos, crianza, papás, estilos.

\section{PARENTS' PERCEPTION ABOUT CHILD REARING}

\begin{abstract}
The aim of the study was to develop a scale to assess parenting styles used by mothers and fathers. The sample was nonprobabilistic and consisted of 790 participants, 350 mothers and 440 fathers of the city of Mexico, with an average age of 36.34 years. The age of the children ranged from 6 to 12 years. A Likert scale with six response options (strongly disagree-strongly agree) was developed. Participants responded the scale in a voluntary manner. After performing the psychometric analysis of the items (bias, total item correlation, Student's $t$ test for extreme groups, factorial analysis of main components with orthogonal rotation, Cronbach's alpha) the scale was composed of 24 items divided into five factors: punishment, permissive, negative emotional, behavioral control, and negative cognition. It was concluded that a valid and reliable scale was obtained for assessing parenting styles used by mothers and fathers.

Key words: children, child rearing, parents, styles.
\end{abstract}

\section{A PERCEPÇÃO DOS PAIS SOBRE A CRIAÇÃO DOS FILHOS}

\author{
Resumo
}

\begin{abstract}
O objetivo do estudo foi elaborar uma escala para avaliar os estilos de criação utilizados por pais e mães. A mostra foi não probabilística e estevo formada por 790 participantes, 350 pais e 440 mães da cidade do México, com uma média de idade de 36.34 anos. A idade dos filhos foi de 6 a 12 anos. Elaborou-se uma escala Likert com seis opções de resposta (totalmente em desacordo-totalmente de acordo). Os participantes responderam a escala de maneira voluntária. Depois de realizar a análise psicométrica aos reativos (desvio, correlação item total, $t$ de Student para grupos extremos, análise fatorial de componentes principais com rotação ortogonal, alpha de Cronbach) a escala ficou integrada por 24 reativos distribuídos em cinco fatores: castigo, permissivo, emocional negativo, controle conductual, e cognição negativa. Conclui-se que se obteve uma escala válida e confiável para avaliar os estilos de criação empregados por pais e mães.

Palavras chave: filos, criação, pais, estilos.
\end{abstract}

* Av Guelato No. 66. Edif. de Gobierno planta baja, Carrera de Psicología. Colonia Ejercito de Oriente. México, D. F. C.P. 09230. mina@unam.mx

Investigación realizada gracias al financiamiento del Programa UNAM-DGAPA-PAPIIT, proyecto clave IN304712. 
En los últimos 25 años las investigaciones sobre familia han evaluado la influencia de diferentes estilos y prácticas parentales, conceptos que frecuentemente los investigadores usan de manera intercambiable. Sin embargo, para entender el proceso de socialización es importante distinguir el uno del otro. Los estilos se refieren al clima emocional en el cual los padres crían a sus hijos; son una actividad compleja que implica comportamientos específicos que trabajan individualmente y en conjunto para influir en los resultados del niño; las prácticas son conductas específicas que los padres utilizan para socializar a sus hijos (Darling, 1999; Darling \& Steinberg, 1993).

En la mayoría de los padres se asume que los estilos de crianza giran en torno a los temas de control, difieren en la manera de controlar o socializar a sus hijos y en el grado en que lo hacen (Baumrind, 1991). Dos formas de control son el psicológico y el conductual. El control psicológico es nocivo debido a que se asocia con problemas emocionales y de conducta de los hijos; los padres intentan controlar el desarrollo psicológico y emocional del niño: sus pensamientos, expresiones y emociones. El control conductual puede ser positivo cuando se utiliza el razonamiento por parte de los papás y mamás en el manejo de los niños (Barber, 1996; Betancourt \& Andrade, 2011).

Sears, Maccoby y Levin (1957) documentaron patrones y procesos de la crianza de los hijos con 300 entrevistas realizadas con madres. Como resultado de esas entrevistas, clasificaron las técnicas disciplinarias utilizadas como orientadas al amor y orientadas al objeto. Explican que el estilo orientado al amor consistió en el uso materno de la calidez, el elogio y el afecto emocional, y en el retiro de esas manifestaciones de acuerdo con los comportamientos de sus hijos. El estilo orientado al objeto radicó en el uso materno de las cosas tangibles (juguetes o tiempo de juego extra), y en el retiro de esos objetos como una forma de responder a la conducta de sus hijos. Los hallazgos de su investigación refieren que los niños de padres que utilizaron el estilo orientado al amor fueron más propensos a interiorizar los valores de sus padres, los cuales asumían como propios, a diferencia de los niños de padres que utilizaban un estilo orientado a los objetos.

Las prácticas utilizadas por los padres, orientadas al objeto o al amor, influyen en la construcción de los estilos de crianza que se integran por un conjunto de conductas parentales encaminadas a crear un clima de interacción dominante sobre una amplia gama de contextos y situaciones (Coplan, Hastings, Lagacé-Séguin, \& Moulton, 2002; Spera, 2005). En los estilos, interviene la capacidad de respuesta, también conocida como calidez o apoyo de los padres. Su importancia radica en la manera en que los padres fomentan la individualidad, la auto-regulación y la aserción; en el opuesto, la exigencia parental alude al control conductual (Maccoby \& Martin, 1983).

A partir del nivel de exigencia y la capacidad de respuesta, se constituye una tipología de cuatro estilos parentales: autoritario, democrático, indulgente e inconsistente. Cada uno de estos estilos refleja diferentes patrones de valores parentales, prácticas y comportamientos que ocurren de manera natural y que distinguen el balance de respuesta y exigencia. El estilo indulgente también se conoce como permisivo; y el inconsistente, como negligente. Los cuatro estilos proveen una constelación de conductas parentales caracterizadas como calidez, exigencia paterna y autonomía otorgada. El estilo autoritario se caracteriza por una baja sensibilidad, alta exigencia y poca autonomía otorgada; el democrático, por alta sensibilidad, alta exigencia paterna y alta autonomía otorgada; el permisivo, muestra altos niveles de sensibilidad y autonomía otorgada y bajos niveles de exigencia paterna; el negligente muestra poco compromiso, bajos niveles de calidez, exigencia y autonomía otorgada (Domenech, Donovick \& Crowley, 2009; Steinberg, Lamborn, Dornbusch \& Darling, 1992).

El estilo de crianza autoritario implica poder de aserción sin calidez, ni cuidado o comunicación (García \& Gracia, 2009). Los padres autoritarios intentan controlar las conductas y actitudes de los hijos con un conjunto absoluto de normas; valoran la obediencia, el respeto a la autoridad y la preservación del orden; carecen de sensibilidad hacia sus hijos, a los que demandan una alta madurez debido a que son intolerantes ante comportamientos inapropiados (Mayseless, Scharf \& Sholt, 2003). Son padres estrictos, pueden ser altamente intrusivos, esperan obediencia a sus órdenes sin ninguna explicación y reafirman el poder cuando sus hijos se portan mal; ofrecen ambientes ordenados y estructurados, con reglas claramente definidas (Baumrind, 1966, 1978; Becker, 1964). Este estilo de crianza es un factor de riesgo para la presencia de timidez y síntomas depresivos en los hijos (Andrade, Betancourt \& Vallejo, 2012; Hummel \& Gross, 2001).

Los padres con autoridad, o democráticos, tienen controles firmes sobre las conductas de sus hijos y hacen fuertes demandas encaminadas a la madurez; son flexibles y están dispuestos a escuchar el punto de vista de sus hijos; frecuentemente se ajustan a los comportamientos de éstos. Combinan su estilo con calidez, cariño, límites firmes, un alto nivel de supervisión, democracia y apertura en la comunicación; escuchan la opinión de los hijos y explican las razones de los castigos (Coplan et al., 2002). Se considera este estilo de crianza como el ideal en la formación de los hijos porque se asocia con una alta autoestima y una baja depresión y ansiedad (DeVores \& Ginsburg, 2005). 
Cuando los padres utilizan un estilo de crianza permisivo, el apoyo es alto y el control es bajo. Es un estilo libre que permite a los hijos regular sus propias actividades con poca interferencia de los padres, debido a que no imponen reglas, solo las enuncian sin consecuencias por su transgresión, situación que permite su fluctuación y transformación constante (Nardone, Giannot \& Rocchi, 2003).

Estos padres son llamados indulgentes o no directivos, evitan la confrontación y es común que cedan a las demandas de sus hijos, quienes tienen mayores probabilidades de presentar problemas académicos y de conducta (Merino \& Arndt, 2004; Snyder, Cramer, Afrank \& Patterson, 2005).

Los padres negligentes muestran poco o ningún compromiso con su rol, no ponen límites a sus hijos porque se carece de interés en hacerlo, son pocas las respuestas afectivas o de control conductual en situaciones diarias y/o en aquellas en que críticamente se requieren. Son padres que puntúan bajo en las dimensiones de exigencia y afectividad; en casos extremos, explícitamente rechazan a sus hijos (Merino \& Arndt, 2004). Al respecto, la teoría de aceptación-rechazo parental, menciona que el rechazo de los padres tiene efectos negativos en la adaptación psicológica y el funcionamiento de la conducta de niños y adultos (Khaleque, Rohner \& Laukkala, 2008), razón por la cual los hijos pueden presentar irritabilidad e inestabilidad emocional (Montts \& Ulloa, 1996).

Para comprender los procesos a través de los cuales los estilos parentales influyen en el desarrollo de los hijos, es importante tener en cuenta tres aspectos de la parentalidad: las metas hacia las cuales se dirige la socialización, las prácticas parentales usadas por los padres para ayudar a los hijos a alcanzar estas metas, y el estilo parental o el clima emocional en el que ocurre la socialización (Darling \& Steinberg, 1993).

En este proceso, para el logro de confianza, autonomía e iniciativa de los niños de 6 a 11 años de edad, es necesario que interactúen con un mayor número de personas para el logro de las metas (Herbert, 2004). Es el periodo en que los profesores, los pares y otras personas externas a la familia tienen mayor contacto con los niños y ejercen diferentes grados de influencia en ellos (Maccoby, 1984).

Para evaluar los estilos y las prácticas parentales se han diseñado instrumentos con diferentes propósitos, para etapas específicas de la niñezy la adolescencia: evaluación de la percepción de los adolescentes referente a los estilos de crianza (Aguilar, Valencia \& Romero, 2004; Márquez-Caraveo, Hernández-Guzmán, Aguilar, Pérez-Barrón \& ReyesSandoval, 2007; Palacios \& Andrade, 2006), evaluación del control parental en niños de 10-13 años (Betancourt \& Andrade, 2007), evaluación de los estilos de socialización parental en niños (Bulnes et al., 2008) y evaluación de las prácticas parentales de crianza en niños (EPPPCN) (Flores, Cortés \& Góngora, 2009).

Además de los instrumentos que evalúan la percepción de los hijos sobre la crianza que ejercen sus padres con ellos, se tienen instrumentos relacionados con la percepción de los padres sobre los estilos que emplean en la crianza de sus hijos. Entre estos se encuentra la escala de autoritarismo en madres con hijos de cero-6 años (Vígano de la Rosa \& Díaz-Loving, 1990); la escala de estilos de crianza para madres con hijos de 3-5 años (Cardona, Nicholson \& Fox, 2000); la escala para madres con hijos de 2-5 años (Robinson, Mandleco, Olsen \& Hart, 1995), y la escala de tolerancia parental aplicada a padres y madres con hijos de 6 a 12 años de edad (Samaniego, 2010).

Son pocos los instrumentos que evalúan la percepción de los padres sobre los estilos que emplean en la crianza de sus hijos, como por ejemplo, la escala de Samaniego (2010) que evalúa la tolerancia parental en Argentina. Con el propósito de evaluar los estilos de manera global, el objetivo de este estudio fue elaborar una escala sobre los estilos de crianza utilizados por papás y mamás con hijos de 6 a 12 años de edad.

\section{MÉTODO}

\section{Participantes}

Se seleccionó de manera no probabilística, una muestra de 790 participantes voluntarios, 350 papás y 440 mamás de la delegación Iztapalapa de la zona oriente de la Ciudad de México. La delegación tiene una superficie territorial de $116.67 \mathrm{~km}^{2}$, que representa el $7.7 \%$ de la superficie del Distrito Federal, por lo que ocupa la cuarta posición de las 16 delegaciones de la ciudad. Reporta el Consejo Nacional de Evaluación de la Política de Desarrollo Social (2012) que es la delegación de mayor población (727,128 habitantes) con un alto índice de marginación social y delincuencia. Ocupa el segundo lugar con personas en condición de pobreza (37.4\%) y de pobreza extrema (3.2\%) (Consejo Nacional de Evaluación de la Política de Desarrollo Social, 2012).

El rango de edad de la muestra fue de 19 a 58 años $(M=36.34, D E=6.98) ; 532$ eran casados $(67.3 \%)$ y 258 convivían en unión libre $(32.7 \%)$. El número de hijos osciló de uno a cinco $(M=2)$. La distribución de los hijos por edades fue: 145 de seis años, 190 de siete años, 167 de ocho años, 156 de nueve años, 184 de 10 años, 169 de 11 años y 81 de 12 años. El número de hijos reportado por los padres fue el siguiente: con un hijo, 148 (18.7\%), con dos, 379 (48.0\%), con tres, 201 (25.4\%), con cuatro, 49 $(6.2 \%)$ y con cinco, $13(1.6 \%)$. 


\section{Instrumento}

El instrumento que se utilizó es resultado de un estudio previo en el que se aplicaron redes semánticas naturales (Reyes-Lagunes \& García, 2008) a una muestra no probabilística de 200 participantes, 100 papás y 100 mamás de la zona oriente de la Ciudad de México, con un rango de edad de 22 a 56 años $(M=35.65, D E=6.74)$. Las redes incluyeron cuatro frases estímulo. Resultado de las redes semánticas y con los planteamientos teóricos sobre los estilos y prácticas parentales, se procedió a elaborar una escala Likert para evaluar la percepción de los padres sobre la forma en que crían a sus hijos.

La escala contiene tres apartados. En el primero se incluye una explicación sobre los objetivos de la investigación y el anonimato de los participantes. En este apartado el participante firma su consentimiento para responder la escala. El segundo apartado está integrado por un grupo de reactivos que tienen como objetivo recabar información de datos sociodemográficos de los participantes. El tercer apartado contiene la escala que se conformó por 68 reactivos distribuidos en seis intervalos de respuestas que van de $1=$ totalmente en desacuerdo a $6=$ totalmente de acuerdo. En la tabla 1 se muestran varios de los reactivos.

Tabla 1.

Reactivos de la escala de estilos de crianza

\begin{tabular}{lccccccc}
\hline & $\begin{array}{l}\text { Totalmente } \\
\text { en desacuerdo }\end{array}$ & & $\begin{array}{r}\text { Totalmente } \\
\text { de acuerdo }\end{array}$ \\
\hline $\begin{array}{l}\text { 6. Permito que mis hijos interrumpan } \\
\text { la plática de los demás }\end{array}$ & 1 & 2 & 3 & 4 & 5 & 6 \\
10. Es complicado educar a mis hijos & 1 & 2 & 3 & 4 & 5 & 6 \\
$\begin{array}{l}\text { 11. Me enojo cuando mis hijos me } \\
\text { desobedecen }\end{array}$ & 1 & 2 & 3 & 4 & 5 & 6 \\
$\begin{array}{l}\text { 14. Me resulta difícil educar a mis hijos } \\
\begin{array}{l}\text { 64. Castigo a mis hijos por no seguir } \\
\text { las reglas establecidas }\end{array}\end{array}$ & 1 & 2 & 3 & 4 & 5 & 6 \\
\hline
\end{tabular}

\section{Procedimiento}

Se acudió a escuelas primarias de la zona oriente de la Ciudad de México, previa autorización de las autoridades del plantel. En cada escuela se reunió a un grupo de papás y mamás con hijos inscritos en los diferentes grados escolares; se les explicó el objetivo de la investigación y se les pidió su colaboración, además de garantizarles la confidencialidad de sus datos. A quienes accedieron a participar se les entregó la escala y se realizó la aplicación de manera grupal. Durante dicha aplicación se respondieron las dudas de los participantes.

\section{RESULTADOS}

Con los datos recabados, se realizó un análisis de los reactivos a través de una distribución de frecuencias, eliminando aquellos que tuvieran un sesgo mayor a $1.500 \mathrm{y}$ una correlación ítem total menor a 0.250 . Con los resultados de la prueba $t$ de Student para grupos extremos, se eliminaron los reactivos 8 y 13 porque su nivel de discriminación fue mayor a 0.05 . A partir de estos criterios, quedaron 33 reactivos a los que se les realizó un análisis factorial de componentes principales con rotación ortogonal. El criterio que se siguió para considerar un reactivo como parte de un factor fue que tuviera un peso factorial superior a 0.40 y que no compartiera peso factorial en otro factor. Como resultado del análisis factorial, la escala quedó integrada por 24 reactivos distribuidos en cinco factores (véase Tabla 2) con valores propios mayores a 1.0 que explican el $50.620 \%$ de la varianza, con un alpha de Cronbach global de .852 .

El factor castigo es una dimensión de los estilos de crianza que comprende prácticas relacionadas con la disciplina de los hijos. Los papás y mamás emplean el regaño y el castigo físico como una forma de corregir a sus hijos. En el factor permisivo, los papás y las mamás tienen poco o ningún control sobre los comportamientos de sus hijos, les permiten autorregularse de acuerdo a sus necesidades e intereses. El factor emocional negativo hace referencia al estado de ánimo de papás y mamás, relacionado con el comportamiento de sus hijos, que ellos consideran inadecuado. Esta percepción les molesta, los frustra, los entristece, los desilusiona y les provoca enojo. El factor control conductual se refiere al otorgamiento de premios de los padres a los hijos cuando cumplen con lo establecido por ellos. El factor cognición negativa es una dimensión que hace referencia a la incertidumbre de papás y mamás relacionada con la manera de criar a sus hijos, la cual les resulta complicada y difícil.

Para obtener las correlaciones entre los factores, se realizó un analisis de modelos estructurales, mediante el programa AMOS (v. 21). El modelo presentó índices de ajuste adecuados: $\mathrm{CFI}=.915, \mathrm{IFI}=.916 \mathrm{y} \mathrm{NFI}=.870 \mathrm{y}$ $\mathrm{RMSA}=.045$. En la figura 1 se presentá la representación gráfica del modelo obtenido.

Los resultados muestran que todas las correlaciones fueron significativas $(\alpha<.001)$. Las correlaciones sugieren que el castigar a los hijos se asocia con la frustración, la tristeza, la desilusión, el malestar y el enojo por su mal comportamiento (emoción negativa) y con la idea de que es difícil y complicado educarlos (cognición negativa). Ante la incertidumbre sobre la crianza de los hijos, la correlación de castigo y permisividad es un indicador de que se les permite a los hijos decidir qué y cuándo hacer lo que les corresponde, 
Tabla 2.

Ponderación de factores con rotación ortogonal de la escala estilos de crianza

\begin{tabular}{|c|c|c|c|c|c|}
\hline \multirow[b]{2}{*}{ Reactivos } & \multicolumn{5}{|c|}{ Factores } \\
\hline & Castigo & Permisivo & $\begin{array}{c}\text { Emocional } \\
\text { negativo }\end{array}$ & $\begin{array}{c}\text { Control } \\
\text { conductual }\end{array}$ & $\begin{array}{c}\text { Cognición } \\
\text { negativa }\end{array}$ \\
\hline 64. Castigo a mis hijos por no seguir las reglas establecidas. & 0.727 & 0.026 & 0.179 & 0.143 & 0.081 \\
\hline 4. Castigo a mis hijos cuando me desobedecen. & 0.651 & -0.039 & 0.051 & 0.108 & 0.257 \\
\hline 37. Les grito a mis hijos por su desobediencia. & 0.616 & 0.183 & 0.224 & -0.005 & 0.213 \\
\hline 51. Cuando mis hijos me desobedecen, les pego. & 0.613 & 0.352 & -0.044 & -0.033 & 0.208 \\
\hline 27. Si mi hijo obtiene bajas calificaciones, lo castigo. & 0.608 & 0.115 & 0.219 & 0.160 & -0.121 \\
\hline 40. Regaño a mis hijos cuando me desobedecen. & 0.590 & -0.073 & 0.341 & 0.035 & 0.037 \\
\hline 54. Dejo que mis hijos haga sus tareas cuando ellos quieran. & -0.031 & 0.709 & 0.021 & 0.056 & -0.096 \\
\hline 58. Permito que mis hijos hagan lo que quieran. & 0.024 & 0.680 & -0.047 & 0.071 & 0.005 \\
\hline 25. Dejo que mis hijos se acuesten a la hora que ellos quieran. & -0.010 & 0.669 & 0.193 & 0.024 & 0.034 \\
\hline $\begin{array}{l}\text { 23. Para que mis hijos dejen de hacer berrinches, cedo a sus } \\
\text { peticiones. }\end{array}$ & 0.171 & 0.599 & -0.013 & 0.108 & 0.130 \\
\hline $\begin{array}{l}\text { 56. A menudo olvido hacer cumplir las reglas que les pongo } \\
\text { a mis hijos. }\end{array}$ & 0.131 & 0.526 & 0.321 & -0.057 & 0.247 \\
\hline 6. Permito que mis hijos interrumpan la plática de los demás. & 0.063 & 0.489 & -0.013 & 0.039 & 0.309 \\
\hline 19. Me entristece que mis hijos se porten mal. & 0.091 & -0.038 & 0.658 & 0.135 & 0.175 \\
\hline 57. Me desilusiona el mal comportamiento de mis hijos. & 0.123 & 0.259 & 0.657 & 0.151 & 0.025 \\
\hline 21. Me molesta que mis hijos no hagan lo que les pido. & 0.270 & -0.021 & 0.623 & 0.080 & 0.144 \\
\hline 43. Me frustra que mis hijos sean maleducados. & 0.234 & 0.245 & 0.558 & 0.163 & 0.061 \\
\hline 11. Me enojo cuando mis hijos me desobedecen. & 0.365 & -0.095 & 0.482 & 0.004 & 0.331 \\
\hline 44. Salimos a pasear cuando mis hijos obedecen. & 0.168 & 0.128 & 0.012 & 0.773 & -0.034 \\
\hline 18. Le doy regalos a mis hijos cuando hacen las cosas bien. & 0.117 & 0.099 & 0.033 & 0.721 & 0.060 \\
\hline 38. Cuando mis hijos me obedecen juego con ellos. & 0.064 & 0.049 & 0.163 & 0.635 & -0.083 \\
\hline 15. Consiento a mis hijos cuando hacen lo correcto. & -0.063 & -0.048 & 0.261 & 0.589 & 0.229 \\
\hline 10. Es complicado educar a mis hijos. & 0.113 & 0.110 & 0.197 & 0.030 & 0.792 \\
\hline 14. Me resulta difícil educar a mis hijos. & 0.214 & 0.139 & 0.166 & 0.027 & 0.753 \\
\hline 45. Se me dificulta que mis hijos sigan las reglas establecidas. & 0.251 & 0.392 & 0.211 & 0.080 & 0.424 \\
\hline Número de reactivos & 6 & 6 & 5 & 4 & 3 \\
\hline Varianza explicada & 23.588 & 9.458 & 7.605 & 5.496 & 4.474 \\
\hline confiabilidad & 0.763 & 0.702 & 0.692 & 0.644 & 0.681 \\
\hline Media & 3.748 & 2.264 & 4.214 & 4.139 & 3.636 \\
\hline
\end{tabular}




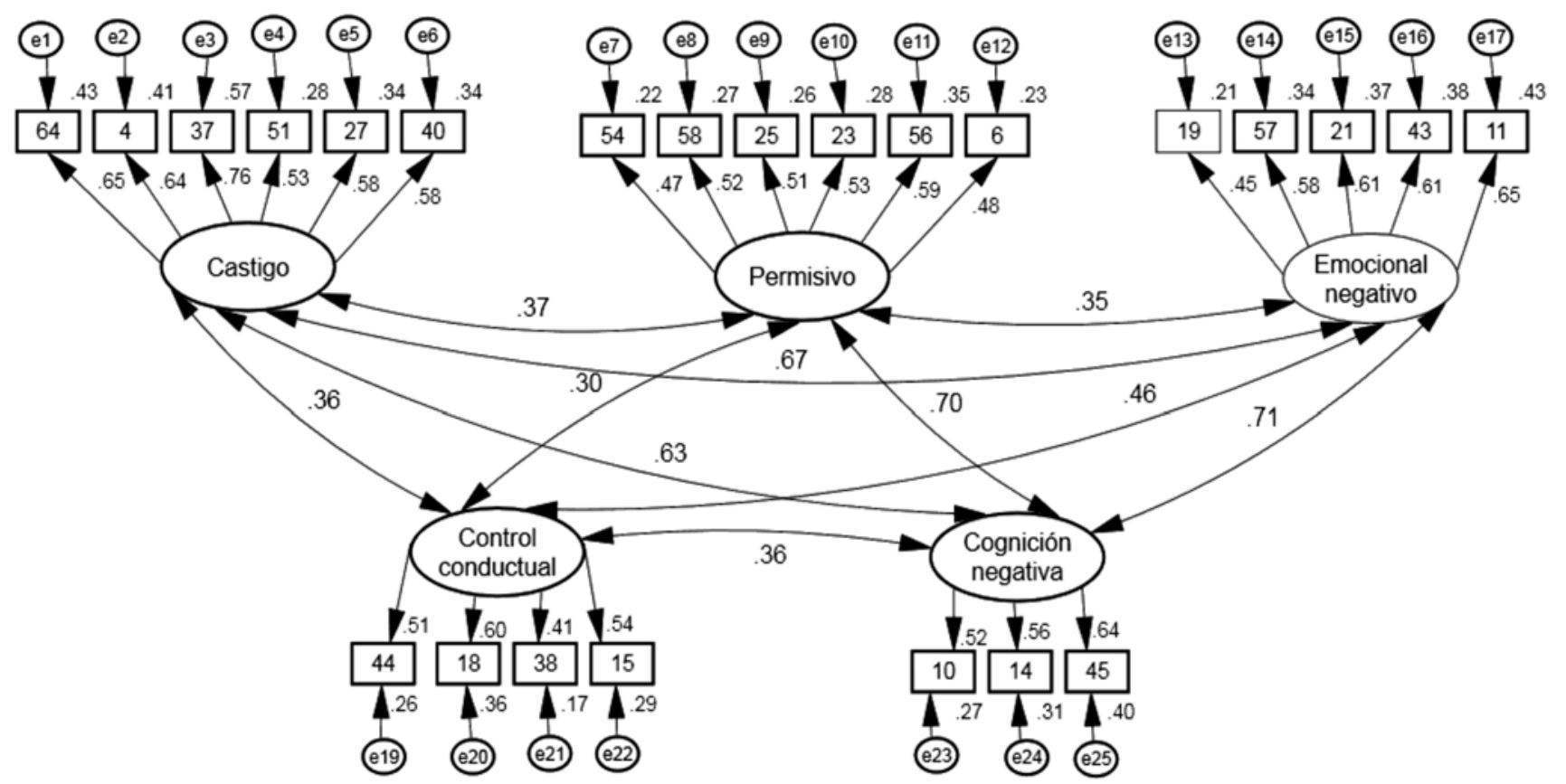

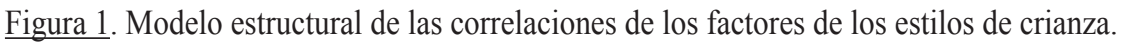

empleando el castigo como una forma de retomar el control sobre su comportamiento, cuando los papás consideran que están rebasando los pocos límites establecidos. Las correlaciones de los factores permisivo, control conductual y cognición negativa, son un indicador que sugiere que los papás premian la autorregulación de los hijos, debido a que les resulta difícil su crianza, otorgándoles recompensas como una forma de controlar su comportamiento.

Con la finalidad de verificar si se mantiene la estructura de la escala, se realizaron cuatro análisis factoriales, dos por rango de edad, a partir de la mediana: grupo $1\left(M_{\text {edad }}\right.$ $=31, D E=3.6)$, grupo $2\left(M_{\text {edad }}=42, D E=4.7\right)$ y dos por sexo. La rotación ortogonal en los cuatro factoriales mantuvo los cinco factores de la escala global, se modificó el número de reactivos, la varianza explicada y los índices de confiabilidad (véase Tabla 3). Debido a limitaciones de espacio, se excluye en este artículo la información sobre la ponderación de factores, resultado de las rotaciones ortogonales de los cuatro análisis.

Tabla 3.

Validez, confiabilidad y número de reactivos de las rotaciones ortogonales

\begin{tabular}{lllll}
\hline & $\begin{array}{l}\text { Grupo 1 } \\
(n=417)\end{array}$ & $\begin{array}{l}\text { Grupo 2 } \\
(n=373)\end{array}$ & $\begin{array}{l}\text { Hombres } \\
(n=350)\end{array}$ & $\begin{array}{l}\text { Mujeres } \\
(n=440)\end{array}$ \\
\hline Varianza explicada & 55.637 & 56.110 & 54.860 & 52.690 \\
Alpha de Cronbach & 0.813 & 0.825 & 0.845 & 0.800 \\
No. de reactivos & 19 & 19 & 22 & 19 \\
\hline
\end{tabular}

Estos resultados sugieren que en México, papás y mamás de diferentes edades emplean los estilos de crianza citados, lo que significa que de acuerdo con la situación y el contexto, utilizarán uno u otro con la finalidad de obtener el mejor beneficio para sus hijos.

Para saber la preferencia de los estilos de crianza utilizados por papás y mamás, se realizó una prueba $t$ de Student para muestras independientes de la escala global (véase Tabla 4).

Las diferencias en castigo, emocional negativo y cognición negativa, sugieren que las mujeres, en contraste con los hombres, utilizan con mayor frecuencia el castigo. La crianza de sus hijos les genera mayor incertidumbre, la perciben como algo difícil, complicado y se enfadan cuando sus hijos no responden a lo establecido por ellas. Esto puede estar asociado a las demandas de su contexto social y cultural, referente a que en la crianza de los hijos, la mujer tiene mayor responsabilidad que el hombre.

Con el propósito de identificar diferencias en el número de hijos y los estilos de crianza, se realizó un ANOVA de un factor, encontrándose que no existían diferencias estadísticamente significativas.

\section{DISCUSIÓN}

Los estilos de crianza son un fenómeno multicausal en el que intervienen diversas variables vinculadas al comportamiento adulto (seguridad, ambivalencia, ansiedad, inseguridad, logro de metas, entre otros) y al bienestar 
Tabla 4.

Diferencias en hombres y mujeres en los estilos de crianza

\begin{tabular}{|c|c|c|c|c|c|c|c|c|}
\hline & \multicolumn{2}{|c|}{ Hombres } & \multicolumn{2}{|c|}{ Mujeres } & \multirow[b]{2}{*}{$t$} & \multirow[b]{2}{*}{$p$} & \multicolumn{2}{|c|}{$95 \% \mathrm{IC}$} \\
\hline & $M$ & $D E$ & $M$ & $D E$ & & & LI & LS \\
\hline Castigo & 3.58 & 1.15 & 3.88 & 1.12 & -3.64 & $<.001$ & -0.46 & -0.14 \\
\hline Permisivo & 2.25 & 1.05 & 2.27 & 1.03 & -.353 & .724 & -0.17 & 0.12 \\
\hline Emocional negativo & 4.04 & 1.15 & 4.35 & 1.11 & -3.83 & $<.001$ & -0.47 & -0.15 \\
\hline Control conductual & 4.08 & 1.19 & 4.18 & 1.13 & -1.19 & .231 & -0.26 & 0.06 \\
\hline Cognición negativa & 3.43 & 1.41 & 3.79 & 1.37 & -3.60 & $<.001$ & -0.56 & -0.16 \\
\hline
\end{tabular}

Nota . IC = Intervalo de Confianza; $\mathrm{LI}=$ Límite inferior; $\mathrm{LS}=$ Límite Superior.

emocional. De tal modo que las cogniciones, actitudes y creencias son factores que influyen en los estilos de crianza empleados por los papás y las mamás. Los factores que conforman la escala reflejan esta influencia.

En el factor castigo, la desobediencia y el no seguir las reglas establecidas, son comportamientos a los que los padres responden con sanciones que implican el regaño y, en ocasiones, el castigo corporal. Este factor se relaciona con el estilo autoritario en el que prevalece la obediencia y el respeto por parte de los hijos, sin cuestionar las órdenes de los padres (Baumrind, 1966; Maccoby \& Martin, 1983). Montts y Ulloa (1996) señalan que los padres cuyas pautas de crianza se caracterizan por los castigos y el maltrato, generan en los hijos mayor irritabilidad e inestabilidad emocional y se potencializa la manifestación de reacciones emocionales exageradas, acompañadas de una falta de control.

En el factor permisivo se agruparon reactivos relacionados con la flexibilidad de la disciplina ejercida por los padres, de tal manera que los hijos pueden normar su comportamiento de acuerdo con lo que ellos consideren conveniente. El compromiso de los padres en la disciplina de los hijos se desvanece y es común que las reglas establecidas no se cumplan. Baumrind (1991) plantea que los permisivos son padres poco demandantes y poco receptivos. Sin embargo, la correlación positiva con el factor castigo sugiere ambivalencia, debido a que se les permite a los hijos determinados comportamientos, pero cuando éstos rebasan los límites de tolerancia de los padres, se ejerce el castigo.

El factor emocional negativo agrupó reactivos asociados con el estado de ánimo de los padres, vinculado al comportamiento de sus hijos. El que sus hijos los desobedezcan les genera enojo, frustración y molestia, condición que tiene una correlación con el castigo, lo cual significa que al aumentar la frustración y el enojo, también se incrementa el castigo hacia los hijos.

En el factor control conductual, los padres otorgan premios solo si los hijos realizan lo establecido por ellos. Sin embargo, esta condición es un elemento que tiene el riesgo de que cuando los hijos no cumplan con las expectativas de sus padres, pueden ser castigados. Al respecto, Becker (1964), Hummel y Gross (2001) señalan que el control es la dimensión de la crianza restrictiva que se caracteriza por un estilo autocrático y de afirmación del poder, relacionado con el uso frecuente de técnicas de castigo-autoritarismo y con la timidez de los hijos.

El factor cognición negativa hace referencia a la percepción que tienen de sí mismos los padres en torno a sus habilidades de crianza. En general, perciben la crianza como difícil y complicada. La correlación de este factor con el castigo sugiere que ante la incompetencia percibida por los padres, sus habilidades para controlar a sus hijos se ven reducidas, lo que puede favorecer el castigo en detrimento del diálogo. En investigaciones sobre el tema, se destaca la importancia de las cogniciones y atribuciones que subyacen a los estilos de crianza que ejercen los papás y las mamás con sus hijos, reportándose una correlación positiva de los padres autoritarios y las cogniciones negativas (García \& Gracia, 2009; Mayseless, Scharf \& Sholt, 2003).

En lo concerniente a las correlaciones de los factores permisivo, emocional negativo y cognición negativa, Snyder et al. (2005) explica, mediante el modelo coercitivo, que las relaciones inseguras entre padres e hijos aumentan la probabilidad de problemas de conducta en los hijos, por lo que una disciplina inconsistente y unas prácticas de crianza rígidas, pueden resultar en conducta antisocial en los hijos.

Por otro lado, el que las mujeres, a diferencia de los hombres, presenten puntajes más elevados en el factor emocional negativo y en las cogniciones negativas, sugiere que se perciben con pocas habilidades y recursos para educar a sus hijos, lo que genera ambivalencia e incertidumbre relacionadas con la manera de interactuar con sus hijos.

Para concluir, resultado de este estudio, con los análisis factoriales por grupo de edad y sexo, y con el modelo de las correlaciones, se confirma la estructura factorial de la escala, obteniéndose un instrumento válido y confiable para evaluar la percepción de los padres relacionada con la crianza de sus hijos. Esta escala contribuirá en la detección 
de factores de riesgo relacionados con comportamientos problemáticos, además de componentes coyunturales que podrán ser empleados para fortalecer las relaciones padreshijos y el proceso de socialización de los niños. La escala podrá ser utilizada en el campo de la psicología clínica y educativa como parte del proceso de detección de problemas, y como un instrumento de investigación relacionado con diferentes variables asociadas con el proceso de formación de los hijos.

\section{REFERENCIAS}

Aguilar, J., Valencia, A. \& Romero, P. (2004). Estilos parentales y desarrollo psicosocial en estudiantes de bachillerato. Revista Mexicana de Psicología, 21(2), 119-129.

Andrade, P., Betancourt, D. \& Vallejo, A. (2012). Prácticas parentales y sintomatología depresiva en adolescentes. Salud Mental, 35, 29-36.

Barber, B. K. (1996). Parental Psychological Control: Revisiting a Neglected Construct. Child Development, 67, 3296-3319.

Baumrind, D. (1966). Effects of authoritative parental control on child behaviour. Child Development, 37(4), 887-907.

Baumrind, D. (1978). Parental disciplinary patterns and social competence in children. Youth and Society, 9(3), 239-276.

Baumrind, D. (1991). The influence of parenting style on adolescent competence and substance use. Journal of Early Adolescence, 11(1), 56-95.

Becker, W.C. (1964). Consequences of different kinds of parental discipline. En M. L. Hoffman \& L. W. Hoffman (Eds.), Review of child development Research (Vol. I pp. 169-208). New York: Russell Sage Foundation.

Betancourt, D. \& Andrade, P. (2007). Escala de percepción de control parental de niños. Ciencias Sociales y Humanidades, 6, 26-34.

Betancourt, D. \& Andrade, P. (2011). Control parental y problemas emocionales y de conducta en adolescentes. Revista Colombiana de Psicología, 20(1), 27-41.

Bulnes, M., Ponce, C., Huerta R., Álvarez, C., Santiváñez, W., Atalaya, M., Aliaga, J. \& Morocho, J. (2008). Resiliencia y estilos de socialización parental en escolares de 4to y 5to año de secundaria de lima metropolitana. Revista de Investigación en Psicología, 11(2), 67-91.

Cardona, P. G., Nicholson, B. C. \& Fox, R. (2000). Parenting among Hispanic and Anglo-american mothers with young children. The Journal of Social Psychology, 140(3), 357-365.

Consejo Nacional de Evaluación de la Política de Desarrollo Social (2012). Informe de pobreza y evaluación en el Distrito Federal 2012. México: CONEVAL.

Coplan, R. J., Hastings, P. D., Lagacé-Séguin, D. G. \& Moulton, C. E. (2002). Authoritative and authoritarian mothers' parenting goals, attributions, and emotions across different childrearing contexts. Parenting: Science and Practice, 2 (1), 1-26.

Darling, N. (1999). Parenting style and its correlates. EEUU: ERIC Digest.

Darling, N. \& Steinberg, L. (1993). Parenting style and context: An integrative model. Psychological Bulletin, 113 (3), 487-496.

DeVores, E. \& Ginsburg, K. (2005). The protective effects of good parenting on adolescents. Current Opinion in Pediatrics 2005, 17(4), 460-465.

Domenech, M. M., Donovick, M. R. \& Crowley, S. (2009). Parenting styles in a cultural context: Observations of "protective parenting" in first-generation latinos. Family Process, 48(2), 195-210.

Flores, M. M., Cortés, M. de L. \& Góngora, E. A. (2009). Desarrollo y validación de la Escala de Percepción de Prácticas Parentales de Crianza para Niños en una muestra mexicana. Revista Iberoamericana de Diagnóstico y Evaluación Psicológica (RIDEP), 2(28), 45-66.

García, F. \& Gracia, E. (2009). Is always authoritative the optimum parenting style? Evidence from Spanish families. Adolescence, 44(173), 101-131.

Herbert, M. (2004). Parenting Across the lifespan. In M. Hoghughi \& N. Long (Eds.), Handbook of Parenting Theory and Research for Practice (pp. 55-71). London: Sage.

Hummel, R. M., \& Gross, A. M. (2001). Socially anxious children: An observational study of parent-child interaction. Child \& Family Behavior Therapy, 23(3), 19-40.

Khaleque, A., Rohner, R. P. \& Laukkala, H. (2008). Intimate partner acceptance, parental acceptance, behavioral control, and psychological adjustment among Finnish adults in ongoing attachment relationships. Cross-Cultural Research, 42, 35-45.

Maccoby, E. E. (1984). Middle childhood in the context of the family. In W. A. Collins (Ed.), Development During Middle childhood, the Years from Six to Twelve (pp. 184-235). Washington DC: National Academy Press.

Maccoby, E. E. \& Martin, J. A. (1983). Socialization in the context of the family: Parent-child interaction. In P. H. Mussen \& E. M. Hetherington (Eds.), Handbook of Child Psychology: Vol. 4, Socialization, Personality, and Development (pp. 1-101). New York: Wiley.

Marquez-Caraveo, M. E., Hernández-Guzmán, L., Aguilar, J., Pérez-Barrón, V. \& Reyes-Sandoval, M. (2007). Datos psicométricos del EMBU-I "Mis memorias de crianza" como indicador de la percepción de crianza en una muestra de adolescentes de la ciudad de México. Salud Mental, 30(2), 58-66.

Mayseless, O., Scharf, M. \& Sholt, M. (2003). From Authoritative Parenting Practices to an Authoritarian Context: Exploring the Person-Environment Fit. Journal of Research on Adolescence, 13(4), 427-456.

Merino, C. \& Arndt, S. (2004). Análisis factorial confirmatorio de la Escala de Estilos de Crianza de Steinberg: Preliminar validez de constructo. Revista de Psicología-PUCP, 12(2), 187- 214. 
Montts, M. \& Ulloa, F. (1996) Autoestima y Salud Mental. Salud Mental, 19(3), 23-30.

Nardone, G., Giannotti, E. \& Rocchi, R. (2003). Modelos de familia. Barcelona: Herder.

Palacios, D. J. \& Andrade, P. P. (2006). Escala de estilos parentales en adolescentes mexicanos. Revista de Psicología Social y Personalidad, 22, 49-64.

Reyes-Lagunes, I. \& García, B. (2008). Hacia un procedimiento de validación psicométrica culturalmente relevante. $L a$ Psicología Social en México, XII, 625-630.

Robinson, C. C., Mandleco, B., Olsen, S. F. \& Hart, C. H. (1995). Authoritative, authoritarian, and permissive parenting practices: Development of a new measure. Psychological Reports, 77(3), 819-830.

Samaniego, V. C. (2010). Escala de tolerancia parental hacia los comportamientos infantiles, elaboración y validación. Revista Latinoamericana de Psicología, 42(2), 203-214.

Sears, R. R., Maccoby, E. E. \& Levin, H. (1957). Patterns of child rearing. Evanston, IL: Row, Peterson.
Snyder, J., Cramer, A., Afrank, J. \& Patterson, G. R. (2005). The contributions of ineffective discipline and parental hostile attributions of child misbehavior to the development of conduct problems at home and school. Developmental Psychology, 41(1), 30-41.

Spera, Ch. (2005). A Review of the relationship among parenting practices, parenting styles, and adolescent school achievement. Educational Psychology Review, 17(2), 125-146.

Steinberg, L., Lamborn, S. D., Dornbusch, S. M. \& Darling, N. (1992). Impact of Parenting Practices on Adolescent Achievement: Authoritative Parenting, School Involvement, and Encouragement to Succeed. Child Development, 63,12661281.

Vígano de la Rosa, D. L. \& Díaz-Loving, R. (1990). Desarrollo y análisis psicométrico del EMACM: Escala multidimensional de autoritarismo en la cultura mexicana. Revista Interamericana de Psicología, 24, 139-158. 